\title{
The effects of viscosity on sound radiation near solid surfaces
}

\author{
C. L. Morfey ${ }^{1}$, S. V. Sorokin ${ }^{2}$ and G. Gabard ${ }^{1} \dagger$ \\ ${ }^{1}$ Institute of Sound and Vibration Research, University of Southampton, Southampton SO17 1BJ, UK \\ 2 Department of Mechanical and Manufacturing Engineering, Aalborg University, \\ 9220 Aalborg, Denmark
}

(Received 25 April 2011; revised 25 August 2011; accepted 11 October 2011; first published online 1 December 2011)

\begin{abstract}
Although the acoustic analogy developed by Lighthill, Curle, and Ffowcs Williams and Hawkings for sound generation by unsteady flow past solid surfaces is formally exact, it has become accepted practice in aeroacoustics to use an approximate version in which viscous quadrupoles are neglected. Here we show that, when sound is radiated by non-rigid surfaces, and the smallest dimension is comparable to or less than the viscous penetration depth, neglect of the viscous-quadrupole term can cause large errors in the sound field. In addition, the interpretation of the viscous quadrupoles as contributing only to sound absorption is shown to be inaccurate. Comparisons are made with the scalar wave equation for linear waves in a viscous fluid, which is extended using generalized functions to describe the effects of solid surfaces. Results are also presented for two model problems, one in a half-space and one with simple cylindrical geometry, for which analytical solutions are available.
\end{abstract}

Key words: aeroacoustics, flow-structure interactions

\section{Introduction}

Viscosity plays a fundamental role in the interactions between a fluid and a moving object, in particular by coupling vorticity and sound waves at the surface. Despite this effect being predominant only for relatively small length scales, it is seen in a variety of real-world applications. For instance, in the cochlea, viscosity is crucial in describing the coupling between the sound waves in the fluid-filled cochlear duct and the travelling waves supported by the basilar membrane. Other examples include micro-actuators or sensors such as microelectromechanical microphones. The present paper aims to clarify the role of viscosity in the flow-acoustic coupling at a material surface, and the framework of acoustic analogies is adapted for this purpose.

The acoustic analogy formulation for sound generation by unsteady flows interacting with solid surfaces was developed by Curle (1955) following Lighthill (1952), and generalized by Ffowcs Williams \& Hawkings (1969). These formulations are exact, but they involve a volume integral in which the viscous stress $\tau$ is convolved with derivatives of a Green's function over the fluid domain $V$, and this 'viscousquadrupole' contribution is typically neglected in aeroacoustic applications (Crighton 1975). Here we re-examine the validity of neglecting the viscous-quadrupole volume

†Email address for correspondence: g.gabard@soton.ac.uk 
term, with particular reference to flows near solid boundaries. Although the analysis will be presented for Curle's analogy (that is, for a fixed control surface), the conclusions apply equally to Ffowcs Williams and Hawkings' formulation for moving boundaries.

We argue below that, since $\tau$ is linearly related to the fluid velocity gradients, part of the viscous-quadrupole 'source term' represents sound attenuation rather than sound generation, and should therefore appear in the wave operator. However, the remainder should be retained, since it may be needed to account for coupling between acoustic and vortical motion. To that end, one should ideally reformulate the analogy using a viscous wave operator, as was suggested by Pierce (1989).

To demonstrate the advantages of such an approach, we carry out the reformulation for the restricted case of small perturbations from a uniform state of rest, and follow Curle (1955) by assuming a fixed (but permeable) control surface as the boundary of $V$. This allows the issue of viscous quadrupoles to be clearly highlighted, and our conclusion (that they can be important under certain defined conditions) will remain valid in more general situations where moving boundaries and nonlinear phenomena are present. Further useful simplification is achieved by assuming a viscous fluid that has zero thermal expansivity; in this special case the thermal and acoustic modes are completely decoupled (Pierce 1989). The result is a viscous linearized wave equation, valid for fluids of arbitrarily large viscosity, that describes the pressure perturbation $p$ at any point in $V$ in terms of $p, v_{n}$ and $\omega$ on a fixed control surface $S$; here $\boldsymbol{\omega}=\operatorname{curl}(\boldsymbol{v})$ is the vorticity, and $v_{n}$ is the fluid velocity normal to $S$.

As a first example, we solve in closed form the problem of a plane boundary with tangential oscillations radiating into a viscous fluid. The solution is used to provide the boundary data required by the viscous wave equation formulation, or by the Curle-Lighthill formulation with the volume term omitted. Without this omission, the two formulations are equivalent; the error resulting from the omission therefore indicates the size of the volume-term contribution to the sound field. Since the contribution depends on the choice of Green's function, the error likewise depends on this choice, as will be discussed further in $\S 4$.

We then present results for the linearized model problem of a circular cylinder whose surface oscillates both normally and tangentially in a viscous fluid. This is a generalization of the corresponding inviscid problem of classical acoustics (Morse \& Ingard 1968), where only the normal surface velocity couples to sound radiation. Note that it includes as a special case the transverse oscillation of a rigid cylinder. As in the plane case, the unsteady pressure is calculated in three ways: (i) by using the Curle surface terms alone, with input data (normal velocity, pressure and viscous stress at the cylinder surface) provided from the analytical solution; (ii) by using the alternative viscous-analogy formulation with input data (normal velocity, pressure and vorticity at the surface) again provided from the analytical solution; and (iii) directly from the exact analytical solution. Since formulation (ii) is also exact, comparison of (ii) and (iii) provides a consistency check. Formulation (i) is approximate, so comparison of (i) and (iii) will demonstrate the effect of neglecting viscous quadrupoles. In what follows we refer to (i) as the Curle surface term (CST) approximation.

The paper begins, in $\S \S 2$ and 3 , with a theoretical analysis of the Curle-Lighthill analogy and a comparison of its source terms with those of the viscous wave equation, under the special conditions outlined above. Several issues with the commonly accepted simplification of the Curle-Lighthill analogy are identified. In $\S \S 4$ and 5 we introduce the two model problems, and identify the region of parameter space 


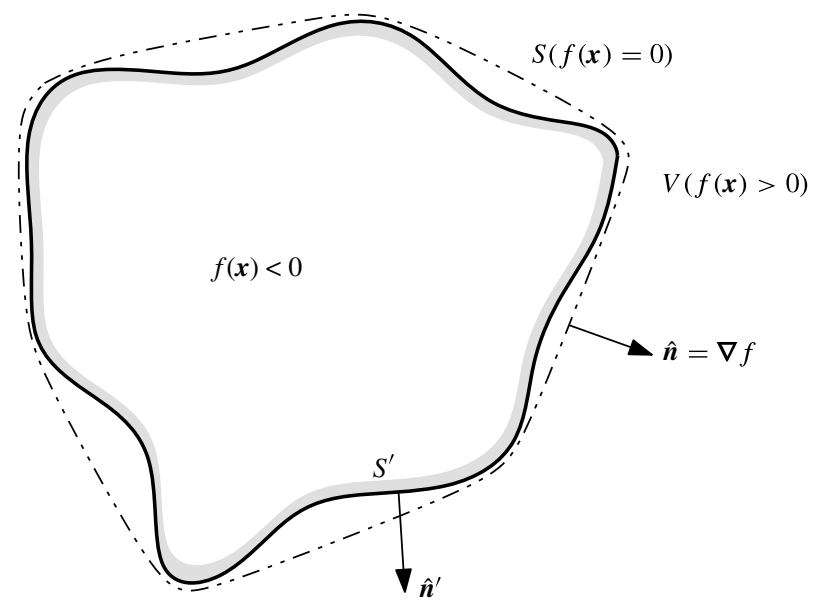

FIGURE 1. Definition sketch showing a vibrating solid object, with perturbed material surface $S^{\prime}$, surrounded by a uniform viscous compressible fluid. The fixed control surface $S$ lies in the fluid, a small distance from the unperturbed solid boundary. Region $V$ lies outside $S$.

where the CST approximation leads to large errors. The principal conclusions are summarized in $\S 6$. An energy corollary for viscous fluids is discussed in appendix A.

\section{Problem definition and notation}

We consider a vibrating, impermeable object surrounded by a volume of fluid $V$, as shown in figure 1 . Let $S$ be a fixed control surface that just encloses the object. To describe the acoustic sources on this surface, we will use a system of orthogonal curvilinear coordinates $y_{i}$. The local orthogonal basis is denoted by the unit vectors $\boldsymbol{e}_{i}$. We identify $\boldsymbol{e}_{3}$ with the normal vector $\hat{\boldsymbol{n}}$, so the vectors $\boldsymbol{e}_{1}$ and $\boldsymbol{e}_{2}$ are tangential to the surface. To avoid ambiguities, we use grad, div, curl and $\Delta$ to denote the gradient, divergence, curl and Laplacian differential operators. The reader can refer to Irgens (2008, chapter 12) for more details.

To perform the analysis presented in this paper, we make use of generalized functions (Farassat 1994) and define the customary indicator function $f$ such that

$$
\begin{array}{ll}
f(\boldsymbol{x})>0 & \text { for } \boldsymbol{x} \in V, \\
f(\boldsymbol{x})<0 & \text { for } \boldsymbol{x} \notin V,
\end{array}
$$

with the additional requirement that, on the surface, $|\operatorname{grad}[f(\boldsymbol{x})]|=1$ so that we can write $\hat{\boldsymbol{n}}=\operatorname{grad}(f)$ for the unit vector normal to $S$. Note that this vector is pointing into the fluid. The Heaviside function $H(f)$ will be used as a spatial windowing function in the equations that follow. Thus we have $H(f)=1$ in region $V$, and $H(f)=0$ elsewhere. In addition, the gradient of $H(f)$ is given by

$$
\operatorname{grad}[H(f)]=\hat{\boldsymbol{n}} \delta(f),
$$

where $\delta$ is the Dirac delta function.

\section{Sound generation in a viscous fluid}

We consider arbitrary small-amplitude oscillations of the material surface $S^{\prime}$ (figure 1) in a uniform ambient fluid, and therefore use the Navier-Stokes equations 
in linearized form. The restriction to small perturbations is not an issue for present purposes, since the volume quadrupole term whose effect we are investigating is linear in the perturbation variables. In addition, to facilitate the discussion, we assume that the thermal expansivity of the fluid is negligible so that acoustic waves in the fluid are decoupled from entropy waves. The linearized relation between pressure, density and entropy perturbations then reduces to $\left(P-P_{0}\right)=c_{0}^{2}\left(\rho-\rho_{0}\right)$, where $c$ is the sound speed and subscript 0 denotes the unperturbed state. In what follows, we write the pressure perturbation $P-P_{0}$ as $p$. The linearized equations of mass and momentum conservation governing the propagation of linear acoustic waves in a viscous fluid at rest can then be written as

$$
\begin{gathered}
\frac{1}{c_{0}^{2}} \frac{\partial p}{\partial t}+\rho_{0} \Theta=0, \\
\rho_{0} \frac{\partial \boldsymbol{v}}{\partial t}+\operatorname{grad}(p)-\operatorname{div}(\boldsymbol{\tau})=\mathbf{0} .
\end{gathered}
$$

Here $\Theta=\operatorname{div}(\boldsymbol{v})$ is the dilatation rate. Finally, if the bulk and shear viscosity are denoted by $\mu_{v}$ and $\mu$, the viscous stress tensor is

$$
\boldsymbol{\tau}=\mu\left(\boldsymbol{L}+\boldsymbol{L}^{\mathrm{T}}\right)+\left(\mu_{v}-2 \mu / 3\right) \Theta \mathbf{1},
$$

with $\boldsymbol{L}=\operatorname{grad}(\boldsymbol{v})$ and 1 indicating the identity tensor. Assuming that $\mu$ and $\mu_{v}$ are constant, we write the divergence of the viscous stress tensor in terms of $\Theta$ and the fluid vorticity $\omega=\operatorname{curl}(\boldsymbol{v})$ :

$$
\operatorname{div}(\boldsymbol{\tau})=\left(\mu_{v}+4 \mu / 3\right) \operatorname{grad}(\Theta)-\mu \operatorname{curl}(\boldsymbol{\omega}) .
$$

\subsection{Viscous wave equation}

Equations (3.1), (3.2) and (3.4) can be combined to obtain a scalar wave equation for small pressure perturbations in a viscous fluid,

$$
\frac{1}{c_{0}^{2}} \frac{\partial^{2} p}{\partial t^{2}}-(1+R) \Delta p=0,
$$

where the operator $R$ accounts for the viscous damping of acoustic waves as they propagate through the fluid,

$$
R=\frac{\mu_{v}+4 \mu / 3}{\rho_{0} c_{0}^{2}} \frac{\partial}{\partial t} .
$$

We now apply the spatial windowing procedure outlined in $\S 2$ to obtain a generalized wave equation for the case with fixed boundaries

$$
\begin{aligned}
& {\left[\frac{1}{c_{0}^{2}} \frac{\partial^{2}}{\partial t^{2}}-(1+R) \Delta\right](H p)} \\
& \quad=\rho_{0} \frac{\partial \boldsymbol{v}}{\partial t} \cdot \hat{\boldsymbol{n}} \delta(f)+\operatorname{div}[-(1+R) p \hat{\boldsymbol{n}} \delta(f)+\mu \boldsymbol{\omega} \times \hat{\boldsymbol{n}} \delta(f)] .
\end{aligned}
$$

Note that in deriving this equation use has been made of the vector identity $\operatorname{div}(\boldsymbol{\omega} \times \boldsymbol{a})=\boldsymbol{a} \cdot \operatorname{curl}(\boldsymbol{\omega})-\boldsymbol{\omega} \cdot \operatorname{curl}(\boldsymbol{a})$ together with the fact that the vector $\boldsymbol{a}=\hat{\boldsymbol{n}} \delta(n)=$ $\operatorname{grad}[H(n)]$ has zero curl, to show that $\mu \operatorname{curl}(\boldsymbol{\omega}) \cdot \hat{\boldsymbol{n}} \delta(n)=\operatorname{div}[\mu \boldsymbol{\omega} \times \hat{\boldsymbol{n}} \delta(n)]$.

In this equation the first term on the right-hand side is the standard monopole source associated with the unsteady volume displacement of the fluid. The second term corresponds to a dipole distribution on the surface $S$. The strength of the normal 
dipoles is governed by the surface pressure fluctuations (modified by the viscous effects $R$ ) while the distribution of tangential dipoles is defined by the tangential vorticity of the fluid on the surface. The latter term causes the sound field to be coupled with the vorticity at the surface. This coupling has already been identified by Pierce (1989) in the limit of weak viscosity; (3.7) represents an extension of Pierce's result, since we do not assume the viscosity to be small.

It is worth noting that (3.7) is in effect a generalization of Kirchhoff's integral. It shows that, to represent the acoustic field in a volume $V$ containing a viscous fluid, one needs to know not only the pressure and normal velocity on the boundary surface $S$ (as is the case with Kirchhoff's integral), but also the tangential vorticity.

\subsection{Curle-Lighthill analogy}

We now consider the Curle-Lighthill analogy (Lighthill 1952; Curle 1955), which, seemingly, leads to a different interpretation of the surface source terms. These differences will be discussed and reconciled in the next section. The Curle-Lighthill analogy in its exact form can be written as

$$
\begin{aligned}
\left(\frac{\partial^{2}}{\partial t^{2}}-c_{0}^{2} \Delta\right)\left[H\left(\rho-\rho_{0}\right)\right]= & \operatorname{div}\left\{\operatorname{div}\left\{H \rho \boldsymbol{v} \otimes \boldsymbol{v}+H\left[p-c_{0}^{2}\left(\rho-\rho_{0}\right)\right] \boldsymbol{1}-H \boldsymbol{\tau}\right\}\right\} \\
& +\frac{\partial}{\partial t}[\rho \boldsymbol{v} \cdot \hat{\boldsymbol{n}} \delta(f)]+\operatorname{div}[-p \hat{\boldsymbol{n}} \delta(f)+\hat{\boldsymbol{n}} \cdot \boldsymbol{\tau} \delta(f)],
\end{aligned}
$$

for a fluid region with fixed boundaries $S$. By using the same assumptions as above, i.e. small perturbations of a viscous fluid at rest with zero expansivity, only the viscous stresses remain in the volume source term and the analogy can be written in terms of the pressure perturbation:

$$
\left(\frac{1}{c_{0}^{2}} \frac{\partial^{2}}{\partial t^{2}}-\Delta\right)(H p)=-\operatorname{div}[\operatorname{div}(H \boldsymbol{\tau})]+\rho_{0} \frac{\partial \boldsymbol{v}}{\partial t} \cdot \hat{\boldsymbol{n}} \delta(f)+\operatorname{div}[t \delta(f)],
$$

where we have introduced the unsteady traction $\boldsymbol{t}=-p \hat{\boldsymbol{n}}+\hat{\boldsymbol{n}} \cdot \boldsymbol{\tau}$ on the surface $S$. It is customary to neglect the double divergence of the windowed viscous stress (the first term on the right-hand side of (3.9)) on the basis that this term represents viscous absorption of sound inside the fluid - see, for instance, Lighthill (1952, p. 568) or Goldstein (1976, p. 71). In other words, it is generally assumed that this term plays the same role as the term $R \Delta$ on the left-hand side of (3.7), which leads to the identification of the surface traction as the exact surface dipole source. We shall show below that this is not generally correct, and leads to a misinterpretation of the surface sources.

\subsection{Discussion of the differences}

We now compare the surface source terms found in (3.9) with the corresponding terms in (3.7). The second term on the right-hand side of (3.9) is the same monopole source as in (3.7). The last term of (3.9) is a dipole distribution entirely defined by the unsteady surface traction. To gain further insight into this dipole source, we can use the definition (3.3) and after some algebra we can write

$$
\begin{aligned}
\boldsymbol{t} & =-(1+R) p \hat{\boldsymbol{n}}+\mu \boldsymbol{\omega} \times \hat{\boldsymbol{n}}-2 \mu(\Theta \hat{\boldsymbol{n}}-\hat{\boldsymbol{n}} \cdot \boldsymbol{L}) \\
& =-(1+R) p \hat{\boldsymbol{n}}+\mu \boldsymbol{\omega} \times \hat{\boldsymbol{n}}-2 \mu\left[\operatorname{div}_{\|}(\boldsymbol{v}) \hat{\boldsymbol{n}}+\operatorname{curl}_{\|}(\boldsymbol{v}) \times \hat{\boldsymbol{n}}\right],
\end{aligned}
$$

where $\operatorname{div}_{\|}$and $\operatorname{curl}_{\|}$are the divergence and curl operators confined to directions tangential to the surface; for a full definition see (B 3). The derivation of (3.10) is not 
trivial and is detailed in appendix B.1. Comparison of (3.10) with the corresponding group of terms in (3.7) indicates that the Curle-Lighthill analogy includes an additional viscous dipole term $-2 \mu\left[\operatorname{div}_{\|}(\boldsymbol{v}) \hat{\boldsymbol{n}}+\operatorname{curl}_{\|}(\boldsymbol{v}) \times \hat{\boldsymbol{n}}\right]$. It is interesting to note that this term is fully defined by the velocity of the surface and hence vanishes when $\boldsymbol{v}=\mathbf{0}$ on $S$.

To provide some insight into this additional viscous dipole, we can consider the perturbed surface $S^{\prime}$ of the solid object with unit normal $\hat{\boldsymbol{n}}^{\prime}$ at each material point, as illustrated in figure 1 . The tensor $\Theta \mathbf{1}-\boldsymbol{L}$ corresponds to the so-called rate-of-surfacestrain tensor as defined by Dishington (1965); see also Wu, Ma \& Zhou (2006). It describes the kinematics of a small surface element of $S^{\prime}$ with area $\delta s^{\prime}$ (which in the unperturbed state has normal $\hat{\boldsymbol{n}}$ and area $\delta s$ ). For instance, one can show that the linearized rate of surface extension is given by $\partial\left(\delta s^{\prime}\right) / \partial t=\operatorname{div}_{\|}(\boldsymbol{v}) \delta s$, and hence that the additional normal viscous dipole in (3.10) is associated with the tangential dilatation of the fluid adhering to the surface. Furthermore, the rate of change in orientation of the surface element is given by $\partial \hat{\boldsymbol{n}}^{\prime} / \partial t=\operatorname{curl}_{\|}(\boldsymbol{v}) \times \hat{\boldsymbol{n}}$, where $\operatorname{curl}_{\|}(\boldsymbol{v})$ can be interpreted as the angular velocity of the fluid layer attached to $S^{\prime}$. This shows that the tangential dipoles are related to rotation of the surface element.

A key point to recognize is that, despite their apparent differences, the two formulations (3.7) and (3.9) are mathematically equivalent, provided that all the terms, and especially the viscous quadrupoles, are retained. This is a consequence of the following identity (the rather lengthy derivation is outlined in appendix B.2):

$$
\begin{aligned}
\operatorname{div}[\operatorname{div}(H \boldsymbol{\tau})] & =-R \Delta(H p)-\operatorname{div}[2 \mu(\Theta \hat{\boldsymbol{n}}-\hat{\boldsymbol{n}} \cdot \boldsymbol{L}) \delta(f)] \\
& =-R \Delta(H p)-\operatorname{div}\left\{2 \mu\left[\operatorname{div}_{\|}(\boldsymbol{v}) \hat{\boldsymbol{n}}+\operatorname{curl}_{\|}(\boldsymbol{v}) \times \hat{\boldsymbol{n}}\right] \delta(f)\right\},
\end{aligned}
$$

where the $R$ term represents the effect of viscosity on sound propagation (it appears on the left-hand side of (3.7)). The second term is a dipole source distributed on the surface, and corresponds precisely to the difference noted between (3.7) and (3.9). By using (3.11) one can rewrite the Curle-Lighthill analogy (3.9) in the form (3.7); the two models are equivalent, provided all the terms are retained in both equations. Equation (3.11) shows that interpreting $\operatorname{div}[\operatorname{div}(H \tau)]$ as a dissipative term associated only with the effect of viscosity on propagation is not valid, because from the viewpoint of the viscous wave equation this term contains both propagation effects and sound sources associated with the boundary $S$. As a consequence, the unsteady surface traction $\hat{\boldsymbol{n}} \cdot \boldsymbol{\tau}$ does not correctly represent the surface dipoles associated with the viscous wave operator in (3.7).

To indicate further the origin of the problem, one can note that the viscous stress tensor is in effect a differential operator acting on the velocity field:

$$
\boldsymbol{\tau}(\boldsymbol{v})=\mu \operatorname{grad}(\boldsymbol{v})+\mu[\operatorname{grad}(\boldsymbol{v})]^{\mathrm{T}}+\left(\mu_{v}-2 \mu / 3\right) \boldsymbol{1} \operatorname{div}(\boldsymbol{v}) .
$$

Before interpreting the double divergence of $H \tau$, one should move the window $H$ under this operator so that $\boldsymbol{\tau}(\cdot)$ applies to the windowed velocity. This is done as follows:

$$
H \boldsymbol{\tau}(\boldsymbol{v})=\boldsymbol{\tau}(H \boldsymbol{v})-\left[\mu(\boldsymbol{v} \otimes \hat{\boldsymbol{n}}+\hat{\boldsymbol{n}} \otimes \boldsymbol{v})+\left(\mu_{v}-2 \mu / 3\right) \boldsymbol{1}(\boldsymbol{v} \cdot \hat{\boldsymbol{n}})\right] \delta(f) .
$$

One can then proceed to derive (3.11) as outlined in appendix B.2.

\subsection{Choice of Green's function}

An advantage of the two acoustic analogies discussed above is that both the inviscid and viscous wave operators can be relatively easily solved using an appropriate 
Green's function. Since both (3.7) and (3.9) are written in terms of generalized functions and are valid in all space, one can choose a variety of Green's functions, provided that they are causal in the time domain, or that they satisfy the appropriate radiation condition in the frequency domain. As long as the source terms are selfconsistent and appropriate to the wave operator under consideration, the sound field found in $V$ should be independent of the choice of Green's function, as illustrated in Morfey, Powles \& Wright (2011).

On the other hand, if the source terms are approximated in any way, for example by discarding the viscous quadrupoles in the standard formulation, this independence property no longer holds. The source approximation may lead to serious errors with one choice of Green's function, but not with another. In $\$ \S 4$ and 5, we demonstrate this numerically for two model problems by considering either the free-field Green's function or the Neumann Green's function, which has the additional constraint that its normal gradient on $S$ vanishes.

\subsection{Special cases}

There are various special cases where it is possible to discuss the difference between the two formulations in more detail.

First of all, for wave scattering problems where the solid surfaces are fixed, it is clear that the surface source terms in the two formulations are equivalent since the additional viscous dipoles identified in (3.10) are identically zero. This is consistent with the conclusions of Davis \& Nagem (2006), who have validated the Curle-Lighthill analogy (with volume source terms omitted) in the compact limit for the scattering of sound waves by a fixed sphere in a viscous fluid, using the analytical solution as benchmark. The only discrepancy attributable to the omission of volume terms was the absence of viscous attenuation.

Another special case is when the solid surface does not rotate but only deforms tangentially. Such a case was considered by Shariff \& Wang (2005) in order to test the prediction of the Curle-Lighthill analogy that surface shear-stress fluctuations are a source of sound. In this case it can be seen from (3.10) that the only difference between (3.9) and (3.7) is a normal dipole associated with the extension of surface elements. In Shariff \& Wang (2005) a Neumann Green's function was used to solve the Curle-Lighthill radiation problem, leading to good agreement between direct numerical simulations and the surface-term predictions of the analogy. We will revisit this model problem in $\S 4$ and demonstrate that, had a different Green's function been used, significant differences could have been observed.

Finally, it is worth noting that, for an acoustically compact solid boundary in rigid-body oscillation, the additional surface source terms found in (3.10) make no contribution to the far-field pressure. When the surface $S$ is small compared to the acoustic wavelength, it is possible to apply the compact source approximation and represent the surface by its monopole and dipole moment integrals (Pierce 1989). The difference in dipole strength between the two formulations (3.7) and (3.9) is then given by

$$
2 \mu \int_{S}\left[\operatorname{div}_{\|}(\boldsymbol{v}) \hat{\boldsymbol{n}}+\operatorname{curl}_{\|}(\boldsymbol{v}) \times \hat{\boldsymbol{n}}\right] \mathrm{d} S .
$$

For rigid-body motion, the velocity field on $S$ is of the form $\boldsymbol{v}(\boldsymbol{x}, t)=\boldsymbol{V}(t)+\boldsymbol{\Omega}(t) \times$ $\left[\boldsymbol{x}-\boldsymbol{x}_{0}(t)\right]$, where $\boldsymbol{V}$ is the velocity of the centroid $\boldsymbol{x}_{0}(t)$ of the oscillating body and $\boldsymbol{\Omega}$ is its angular velocity. One can show easily that in that case $\operatorname{div}_{\|}(\boldsymbol{v})=0$ and $\operatorname{curl}_{\|}(\boldsymbol{v})=\boldsymbol{\Omega}+(\boldsymbol{\Omega} \cdot \hat{\boldsymbol{n}}) \hat{\boldsymbol{n}}$. It then follows that $\left[\operatorname{curl}_{\|}(\boldsymbol{v})\right] \times \hat{\boldsymbol{n}}=\boldsymbol{\Omega} \times \hat{\boldsymbol{n}}$. Finally Stokes' 
theorem leads to the conclusion that the integral equation (3.14) vanishes:

$$
2 \mu \int_{S}\left[\operatorname{div}_{\|}(\boldsymbol{v}) \hat{\boldsymbol{n}}+\operatorname{curl}_{\|}(\boldsymbol{v}) \times \hat{\boldsymbol{n}}\right] \mathrm{d} S=2 \mu \int_{S} \boldsymbol{\Omega} \times \hat{\boldsymbol{n}} \mathrm{d} S=-2 \mu \int_{V} \operatorname{curl}(\boldsymbol{\Omega}) \mathrm{d} V=0 .
$$

Thus for compact objects in rigid-body motion the two surface dipole distributions in (3.7) and (3.9) are equivalent. Where the motion is purely translational, as for an oscillating sphere (Pierce 1989), the equivalence holds for any Helmholtz number.

\section{Application to an oscillating plane surface}

As a first example, we solve in closed form the problem of a plane boundary with tangential oscillations radiating into a viscous fluid (with the bulk viscosity $\mu_{v}$ set equal to zero). In figure $2(a)$ an oscillating surface at $x_{2}=0$ radiates into the upper half-space filled with a viscous fluid at rest. The surface velocity is purely tangential, and takes the form of a harmonic travelling wave:

$$
v_{1}\left(x_{1}, x_{2}=0, t\right)=V \mathrm{e}^{\mathrm{i} k_{1} x_{1}-\mathrm{i} \omega t}, \quad v_{2}\left(x_{1}, x_{2}=0, t\right)=0 .
$$

This type of excitation is commonly referred to as a 'dolphin-skin' oscillation. The solution depends on two non-dimensional parameters $L=\omega \mu /\left(\rho_{0} c_{0}^{2}\right)$ and $M=\omega /\left(k_{1} c_{0}\right)$, where $M$ is the phase Mach number of the oscillation along the plane boundary. The parameter $L$ is directly related to the operator $R$ introduced in (3.6) for the viscous wave equation. For $\mathrm{e}^{-\mathrm{i} \omega t}$ time-harmonic solutions this operator corresponds to a scalar $\hat{R}=-\mathrm{i} \omega 4 \mu /\left(3 \rho_{0} c_{0}^{2}\right)$ and we have the relation $L=3 \mathrm{i} \hat{R} / 4$.

This problem is similar to that solved using direct numerical simulation by Shariff \& Wang (2005). As shown in appendix C, one can solve the linearized equations (3.1) and (3.4) to obtain closed-form expressions for all quantities of interest. It is then straightforward to evaluate the acoustic pressure field using either the CST approximation - namely (3.9) with the first term on the right omitted - or alternatively the viscous formulation (3.7), and to compare the resulting pressure amplitudes on the boundary with the exact solution. Because (3.7) and (3.9) are valid in all space rather than only in $V$, any causal Green's function for the relevant operator may be used to obtain the correct pressure field (Morfey et al. 2011) provided the full source terms are retained. However, the CST approximation leads to errors that depend on the choice of Green's function, as we demonstrate below.

Using the free-field Green's function, $G_{\infty}$, for the inviscid acoustic wave operator in (3.9) leads to the following expression for the pressure on the oscillating surface:

$$
p_{C S T}\left(x_{1}, x_{2}=0, t\right)=\mathrm{i} k_{1} G_{\infty}\left(0 \mid 0 ; k_{20}\right) \tau+\mathrm{i} k_{20} G_{\infty}\left(0 \mid 0 ; k_{20}\right)(\sigma-p) .
$$

Here $p, \tau$ and $\sigma$ on the right-hand side denote the pressure and the tangential and normal components of the viscous surface traction that are provided by the closedform solution; $G_{\infty}(x \mid y ; \kappa)$ is the one-dimensional free-field Green's function and $k_{20}$ is the transverse wavenumber of the lossless acoustic wave, as defined in appendix $\mathrm{C}$. In (4.2) the first term on the right-hand side represents the tangential shear-stress dipole while the second is the contribution from the normal dipole.

Alternatively, if we use the Neumann Green's function $G_{N}$ with zero normal gradient at the surface, the pressure is given directly by

$$
p_{C S T}\left(x_{1}, x_{2}=0, t\right)=\mathrm{i} k_{1} G_{N}\left(0 \mid 0 ; k_{20}\right) \tau,
$$

with no contribution from the normal dipole.

We can compare the predictions of the CST formulation against the exact closedform solution by plotting $20 \log _{10}\left|p_{C S T} / p_{\text {exact }}\right|_{x_{2}=0}$, which provides a measure of the 
(a)

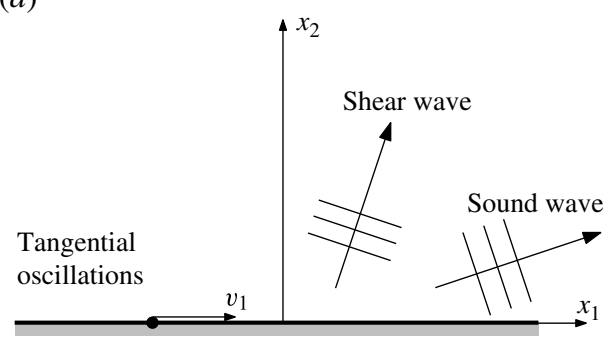

(b)

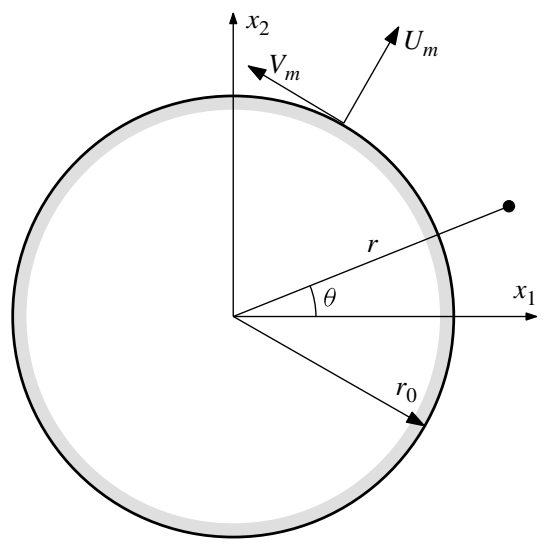

FIGURE 2. (a) A plane surface with tangential oscillations radiating into a half-space.

(b) Definition sketch for the test case of a cylinder in a viscous fluid.

error based on the surface pressure. The results are shown in figure 3 using either the free-field Green's function or the Neumann Green's function, and for a range of parameters $L$ and $M$. Figure 3 shows that, with the free-field Green's function, the error associated with the CST formulation can be large, especially for evanescent waves $(M<1)$, where the pressure amplitude on the cylinder is severely overestimated by the CST formulation and the error increases rapidly with $L$. However, with the Neumann Green's function, only minute differences can be observed. The explanation can be found in (3.10), where the difference between the CST surface source terms (3.9) and those of the exact viscous analogy (3.7) has been identified as a dipole distribution $2 \mu\left[\operatorname{div}_{\|}(\boldsymbol{v}) \hat{\boldsymbol{n}}+\operatorname{curl}_{\|}(\boldsymbol{v}) \times \hat{\boldsymbol{n}}\right]$. For a purely tangential oscillation of the surface, there is no rotation of the surface elements, so $\operatorname{curl}_{\|}(\boldsymbol{v})=0$ and only the normal dipole $2 \mu \operatorname{div}_{\|}(\boldsymbol{v}) \hat{\boldsymbol{n}}$ associated with the extension of the surface elements remains. When using the Neumann Green's function to solve the CST formulation, normal surface dipoles are naturally excluded, and we are left with surface source terms that are consistent with those of (3.7). The small error observed in figure 3 originates from the fact that the inviscid Green's function is used for the CST formulation whereas the viscous wave operator appears on the left-hand side of (3.7). The difference between solving the lossless or viscous wave equations yields only very small errors in the pressure at the plane boundary, but would lead to increasingly larger discrepancies as the observer moves farther away from the boundary.

This indicates that the good agreement observed by Shariff \& Wang (2005) between the CST formulation and their direct numerical simulation relies on the use of the Neumann Green's function. Had the free-field Green's function been used instead, the normal dipole source terms would have been included on the boundary and significant discrepancies might have been observed, mainly in the near field, where the evanescent waves would be strongly overestimated by the CST formulation.

\section{Application to a vibrating cylinder}

\subsection{Problem definition}

To discuss further the issues identified in $\S 3$, we now consider a two-dimensional test case, shown in figure $2(b)$, of a cylinder with radius $r_{0}$ in a viscous fluid at rest with mean density $\rho_{0}$, sound speed $c_{0}$ and shear viscosity $\mu$ (including the bulk viscosity in this test case does not change the conclusions). The surface of the cylinder oscillates 

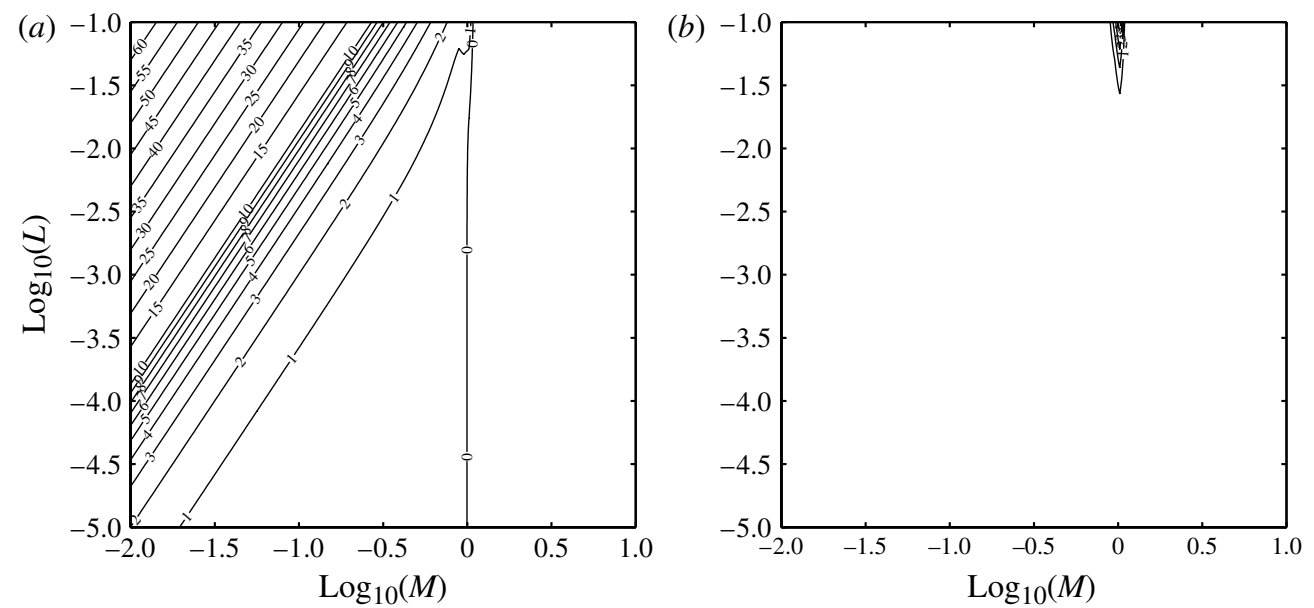

FIgURE 3. Contours of the error in $\mathrm{dB}, 20 \log _{10}\left[\left|p_{C S T} / p\right|\left(x_{1}, x_{2}=0\right)\right]$, associated with the CST formulation for the pressure on a plane boundary. The boundary motion is tangential, as in (4.1). The error depends on the Green's function: $(a)$ free-field Green's function (contour lines from 0 to $10 \mathrm{~dB}$ every $1 \mathrm{~dB}$, and then from 10 to $70 \mathrm{~dB}$ every $5 \mathrm{~dB}$ ); $(b)$ Neumann Green's function (contour lines from 0 to $5 \mathrm{~dB}$ every $1 \mathrm{~dB}$ ).

with time-harmonic normal and tangential velocities given by

$$
U(\theta, t)=U_{m} \cos (m \theta) \mathrm{e}^{-\mathrm{i} \omega t}, \quad V(\theta, t)=V_{m} \sin (m \theta) \mathrm{e}^{-\mathrm{i} \omega t}, \quad \text { with } m=0,1,2, \ldots,
$$

respectively. The corresponding pressure field induced in the fluid by the oscillations of the cylinder can be written as

$$
p(r, \theta, t)=p_{m}(r) \cos (m \theta) \mathrm{e}^{-\mathrm{i} \omega t} .
$$

As shown in appendix $\mathrm{D}$, the complex amplitude $p_{m}$ can be obtained in closed form by solving the linearized Navier-Stokes equations. This exact solution provides a means to validate the predictions obtained from either the viscous wave equation (3.7) or the Curle-Lighthill equation (3.9); in particular, we can examine the effect of omitting the viscous-quadrupole term from (3.9). The response of the fluid to normal $\left(U_{m}\right)$ and tangential $\left(V_{m}\right)$ boundary oscillation is proportional to the respective forcing amplitudes, and also depends on $m$ and two real non-dimensional parameters,

$$
S=\frac{\rho_{0} r_{0}^{2} \omega}{\mu} \quad \text { and } \quad L=\frac{\omega \mu}{\rho_{0} c_{0}^{2}}=\frac{K^{2}}{S},
$$

where the Helmholtz number $K=\omega r_{0} / c_{0}$ relates $r_{0}$ to the lossless acoustic wavelength and the Stokes number $S$ relates $r_{0}$ to the viscous penetration depth.

\subsection{Comparisons of CST predictions with the exact solution}

The predictions of the windowed viscous wave (3.7) are found to be in complete agreement with the closed-form solution for this test case, for all values of $m, L$ and $S$. This is as expected, because (3.7) is a direct consequence of the linearized Navier-Stokes equations used to derive the closed-form solution.

To assess rigorously the issues associated with the CST approximation based on (3.9), we must first recognize that another source of error can play a role. 

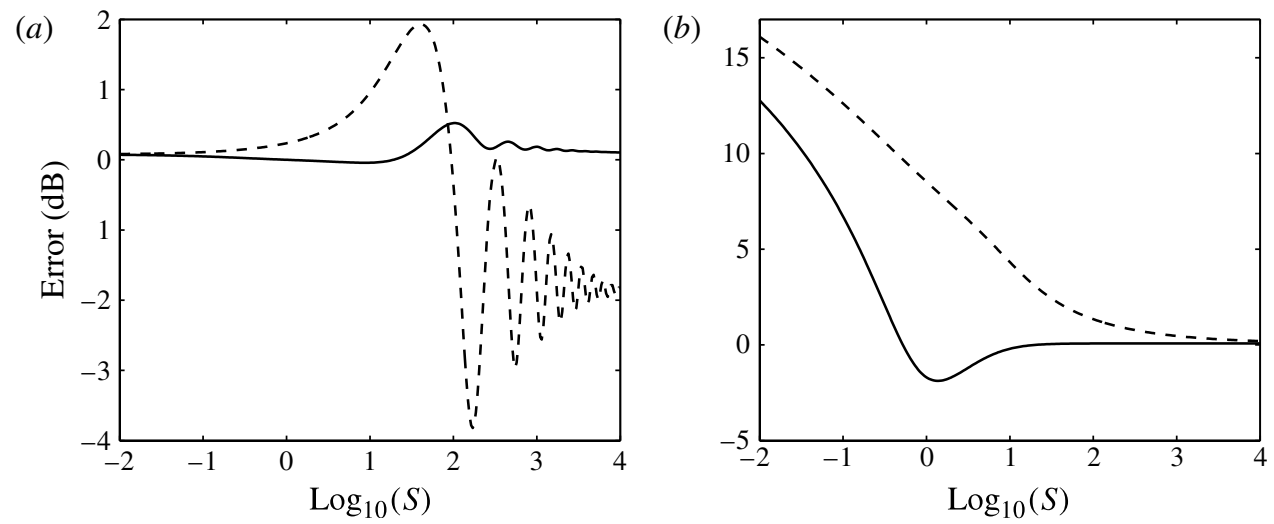

FIGURE 4. Mode $m=1$ with $L=10^{-1}$ : (a) free-field Green's function; (b) Neumann Green's function. Solid line: normal velocity forcing. Dashed line: tangential velocity forcing.

As already mentioned in $\S 4$, the propagation operator in the Curle-Lighthill analogy is that of a lossless fluid, whereas the exact solution includes the viscous damping of waves radiating from the cylinder. To highlight more clearly the differences associated with the surface source terms (rather than the effect of viscosity on propagation), we compare solutions for surface pressure on the cylinder by plotting

$$
20 \log _{10}\left[\left|\hat{p}_{m}\left(r_{0}\right) / p_{m}\left(r_{0}\right)\right|\right],
$$

where $\hat{p}_{m}$ is the Curle-Lighthill solution based on surface terms alone. This eliminates the viscous damping of sound waves as they propagate away from the cylinder. However, it does not completely remove the difference in Green's functions between (3.7) and (3.9) and, as shown in appendix $\mathrm{D}$, a factor $(1+\hat{R})^{-1 / 2}$ can be observed in the limit of low Helmholtz numbers. Finally, since it was shown in $\S 4$ that the choice of Green's function can be significant, we present results for both free-field and Neumann Green's functions.

We begin by highlighting some cases where the CST approximation deviates significantly from the closed-form solution. Figures 4 and 5 show the error measure defined in (5.4), for $m=1$ and two different $L$ values $\left(L=10^{-1}\right.$ and $\left.10^{-5}\right)$. With the free-field Green's function, $G=G_{\infty}$, the peak error is of the order of $10 L^{1 / 2} \mathrm{~dB}$ and thus becomes small for realistic values of $L$. On the other hand, if the Neumann Green's function is used, $G=G_{N}$, then the errors associated with the CST approximation increase without bound as $S \rightarrow 0$, and are relatively insensitive to $L$.

Changing the azimuthal order from $m=1$ to $m=2$ (figure 6) alters the $G_{\infty}$ errors drastically at low Helmholtz numbers $(K=\sqrt{L S}<1)$; they now become comparable to the $G_{N}$ errors for $m=1$, amounting to several decibels at $S=10^{-2}$. On the other hand, the $G_{N}$ errors for $m=2$ show little change from $m=1$.

The marked difference associated with the choice of Green's function in figures 4, 5 and 6 indicates the problem in using the Curle-Lighthill analogy without the $\operatorname{div}[\operatorname{div}(H \boldsymbol{\tau})]$ term. Finally, we mention two special cases where, in contrast, the latter term has no effect on the sound source.

The first such case is that of a rigid body oscillating in translation, which corresponds to $m=1$ and $U_{m}=-V_{m}$. It has been verified that in this case the CST approximation matches the closed-form solution exactly on the cylinder, apart from the 

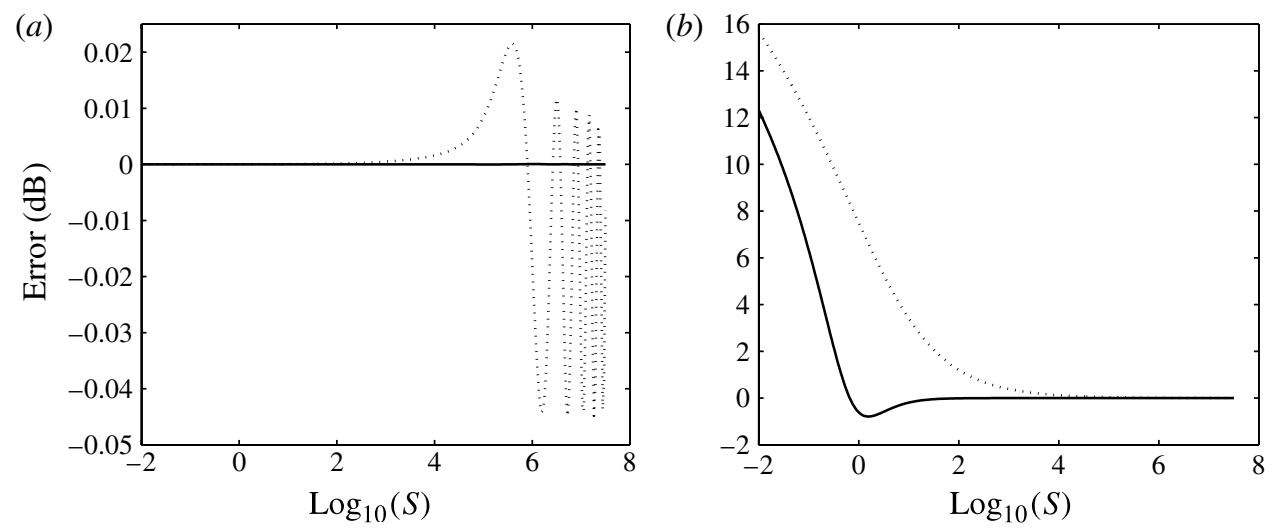

FIGURE 5. Mode $m=1$ with $L=10^{-5}$ : (a) free-field Green's function; (b) Neumann Green's function. Solid line: normal velocity forcing. Dashed line: tangential velocity forcing.
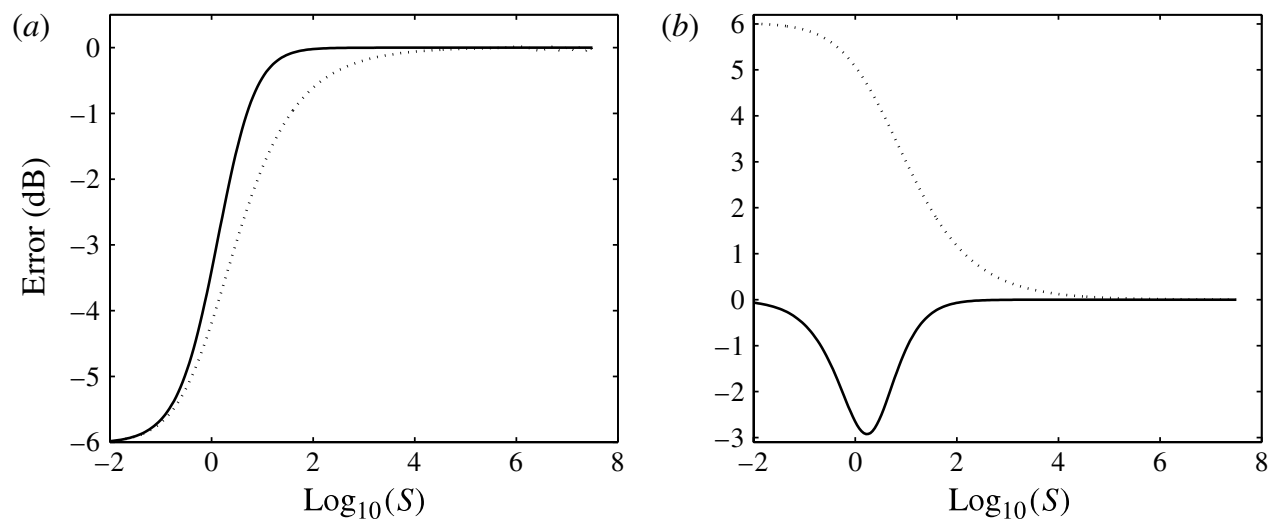

FIgURE 6. Mode $m=2$ with $L=10^{-5}$ : (a) free-field Green's function; ( $b$ ) Neumann Green's function. Solid line: normal velocity forcing. Dashed line: tangential velocity forcing.

factor $(1+\hat{R})^{1 / 2}$ already mentioned and associated with the difference in propagation operators.

The second case is that of a pulsating cylinder corresponding to $m=0, V_{m}=0$ and $U_{m} \neq 0$. In this case the surface elements on the cylinder undergo a time-harmonic extension-contraction but no rotation and therefore the only additional viscous dipole in (3.10) is the normal dipole of strength $-2 \mu \operatorname{div}_{\|}(v)$. For that reason, the use of the Neumann Green's function yields the expected result (again with a $(1+\hat{R})^{1 / 2}$ coefficient) because this Green's function does not involve the normal dipole sources on the surface. With the free-field Green's function, figure 7 shows that there is a small but finite error peaking at $K \approx 1$. At low frequencies $(K \ll 1)$, the error vanishes. This is because the cylinder is then acoustically compact, and the dipole moment integral (3.14) vanishes owing to the fact that $\operatorname{div}_{\|}(v)$ is uniform over the cylinder.

\subsection{Relative sound pressures due to tangential and normal surface oscillation}

The cylinder test case allows one to compare the relative efficiency of normal and tangential oscillations of the surface as a source of sound. This can be measured by 


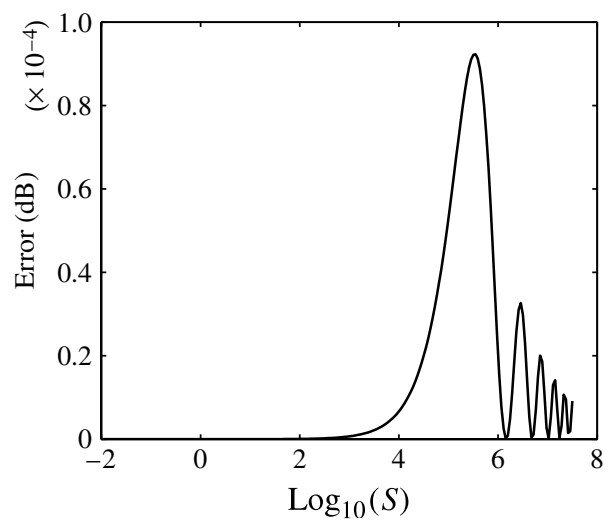

FIGURE 7. Mode $m=0$ with $L=10^{-5}$, using the free-field Green's function.

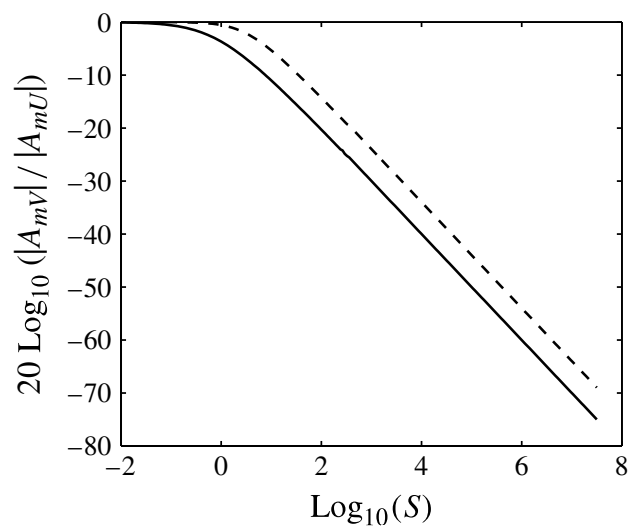

FIGURE 8. Ratio of sound pressure amplitudes between the case of tangential oscillations and normal oscillations, with $L=10^{-5}$ and for $m=1$ (solid line) and $m=2$ (dashed line). Altering $L$ to $10^{-3}$ produces no visible change in the graph.

computing the ratio between the acoustic-wave amplitudes $A_{m}$ given in appendix D for tangential oscillations $\left(U_{m}=0\right.$ and $\left.V_{m}=1\right)$ and normal oscillations $\left(U_{m}=1\right.$ and $\left.V_{m}=0\right)$. The ratio is plotted in figure 8 for $m=1$ and $m=2$. It can be seen that, as expected, for $S>1$ normal oscillations are much more efficient at radiating sound. For $S<1$ the efficiency of tangential oscillations approaches that of normal oscillations. Interestingly, this ratio was found to be virtually independent of $L$.

It is interesting to compare the cylinder results in figure 8 with the corresponding result for the oscillating plane boundary considered in $\S 4$. The ratio in the latter case can be derived explicitly as $\left(\mathrm{i} M^{2} / L-1\right)^{-1 / 2}$. The two cases can be connected by 'unwrapping' the cylinder to form a plane boundary, and equating the phase speed $c_{0} M$ along the plane boundary to $a \omega / \mathrm{m}$. This procedure, asymptotically valid for $S \gg 1$, yields $M^{2} / L=S / \mathrm{m}^{2}$; thus when $S$ is large, the ratio of sound pressure amplitudes should be a function of $S$ alone (for given $m$ ), as is indicated in figure 8 . Note that the amplitude ratio approaches unity for small Stokes numbers $(S<1)$. 


\section{Summary and concluding remarks}

The viscous-quadrupole volume source term found in the acoustic analogy formulations of Lighthill (1952), Curle (1955) and Ffowcs Williams \& Hawkings (1969) has been discussed in detail, with emphasis on sound radiation near boundaries, where the relevant term takes the form $\operatorname{div}[\operatorname{div}(H \boldsymbol{\tau})]$ with $H$ a spatial windowing function (defined in $\S 2$ ). The shear viscosity $\mu$ of the fluid is treated as a constant, but the viscous parameter $\omega \mu / \rho_{0} c_{0}^{2}$ is not required to be small. In the absence of solid surfaces, (3.4) shows directly that $\operatorname{div}[\operatorname{div}(\tau)]=\left(\mu_{v}+4 \mu / 3\right) \Delta \Theta$, indicating that this source depends only on the dilatation rate, and not on the local vorticity. As a consequence, the compact-source argument used to scale multipole sources in Lighthill (1952) does not apply. Moreover, considering each octupole component of the viscous source term independently as in Crighton (1975) does not necessarily reveal the actual nature of this source. In contrast to previous analyses by Lighthill (1952) and Crighton (1975), which were limited to unbounded flows, we find that the role of the viscous volume term is not restricted to sound absorption.

This viscous term can be reformulated, following a suggestion by Pierce (1989), to reveal an extra surface-dipole distribution that supplements the viscous traction dipoles given by Curle's theory (1955). The additional surface-dipole sources become significant at Stokes numbers $S$ of order one or less, as we demonstrate in $\S 5$ for radiation from a vibrating cylindrical surface.

The following principal conclusions can be drawn from the present work.

(a) The role of the viscous-quadrupole volume source term is made apparent by changing from the lossless wave operator used by Lighthill (1952) and Curle (1955) to a viscous wave operator, as in (3.7).

(b) With this change, viscous effects on sound propagation appear on the left-hand side of the wave equation. In an unbounded fluid, with thermal expansivity set equal to zero, the pressure perturbation $p$ satisfies a homogeneous linearized equation at small amplitudes. For a bounded region (with spatial windowing function $H$ ), the linearized equation for $H p$ involves monopole and dipole sources on the boundary $S$, with a dipole source distribution that differs from the one found by Curle (1955).

(c) More specifically, extension or stretching of fluid surface elements on $S$ is responsible for the difference in the normal-dipole component. Likewise, if such fluid elements change their orientation at a finite rate, the tangential-dipole component is different in the two formulations.

(d) The difference in surface-dipole distributions, as between the lossless Curle-Lighthill analogy and the present viscous analogy, vanishes if the fluid velocity is zero on $S$. Thus for problems of wave scattering by rigid surfaces, the two formulations yield identical surface source terms (although the different wave operators imply different Green's functions).

(e) In sound radiation problems characterized by one or both of the features in $(c)$, using the Curle surface terms to estimate the sound field will generally lead to serious errors at low Stokes numbers. This is separate from any propagation effects. Thus when the dimensions of the radiating surface are smaller than the viscous penetration depth, a viscous formulation as in (3.7) is required.

( $f$ ) Because use of the Curle surface terms alone is an approximation, the error in the resulting sound-field estimate will depend on the choice of Green's function. For example, in the case of radiation from 'dolphin-skin' expansions and contractions 
of a vibrating surface, errors in near-field pressure are largely eliminated by choosing the Neumann Green's function whose normal derivative vanishes on $S$ (as demonstrated in figure 3).

\section{Acknowledgement}

This paper is dedicated to A. Pierce, author of Acoustics: An Introduction to its Physical Principles and Applications (1989), to mark his 75th birthday.

\section{Appendix A. Energy balance}

It is also useful to relate the present analysis with the energy balance in the fluid. To that end we apply the scalar product between $\boldsymbol{v}$ and the momentum equation (3.2), and then use the mass equation (3.1) and the identity (3.4) to arrive at an energy balance equation of the form

$$
\frac{\partial e}{\partial t}+\operatorname{div}(\boldsymbol{f})=-d
$$

with the following definitions for the density $e$, flux $f$ and dissipation $d$ of energy:

$$
e=\frac{p^{2}}{2 \rho_{0} c_{0}^{2}}+\frac{1}{2} \rho_{0}|\boldsymbol{v}|^{2}, \quad \boldsymbol{f}=(1+R) p \boldsymbol{v}+\mu \boldsymbol{\omega} \times \boldsymbol{v}, \quad d=\left(\mu_{v}+4 \mu / 3\right) \Theta^{2}+\mu|\boldsymbol{\omega}|^{2} .
$$

These definitions are consistent with those obtained by Pierce (1989). One difference is that, in the case considered here of a fluid with zero expansivity, entropy waves are decoupled from acoustic waves so the terms involving entropy and temperature fluctuations $s$ and $T$ are not involved in the energy balance (A 1) (in fact, one could derive a separate energy balance for the entropy waves). Another difference is the use of (3.4) to separate more clearly the contributions from acoustic waves and vorticity in the energy flux and dissipation.

Note that the energy balance defined by (A 2) is not restricted to acoustic-mode disturbances; for example, the vortical component of $\boldsymbol{v}$ contributes to the second term in each of $e, f$ and $d$. In (A 2) the dissipation of energy is clearly defined as two distinct contributions from the dilatation rate and the enstrophy (defined as $|\omega|^{2}$ ). As for the flux of energy, the contributions of acoustic pressure and vorticity are now apparent. Because both vorticity and sound waves contribute to the velocity field $\boldsymbol{v}$, the flux terms $p \boldsymbol{v}$ and $\boldsymbol{\omega} \times \boldsymbol{v}$ contain cross-terms between vorticity and acoustics. One should not view $(1+R) p v$ as the contribution of acoustic waves alone to the energy flux, and $\mu \omega \times v$ as the contribution of vorticity alone.

The link with the viscous wave analogy (3.7) becomes more obvious when considering the normal flux of energy on a boundary:

$$
\begin{aligned}
\boldsymbol{f} \cdot \hat{\boldsymbol{n}} & =(1+R) p \boldsymbol{v} \cdot \hat{\boldsymbol{n}}+\mu(\boldsymbol{\omega} \times \boldsymbol{v}) \cdot \hat{\boldsymbol{n}} \\
& =[(1+R) p \hat{\boldsymbol{n}}-\mu(\boldsymbol{\omega} \times \hat{\boldsymbol{n}})] \cdot \boldsymbol{v} .
\end{aligned}
$$

In the last expression the two terms in square brackets are precisely the normal and tangential surface dipoles identified in the analogy based on the viscous wave equation (3.7). This underlines again the central role played by these terms in transferring energy from a vibrating surface into the fluid. 


\section{Appendix B. Details of mathematical derivations}

\section{B.1. Derivation of (3.10)}

To derive equation (3.10) we begin by using the definition (3.3) together with the identity $\hat{\boldsymbol{n}} \cdot \boldsymbol{L}^{\mathrm{T}}=\hat{\boldsymbol{n}} \cdot \boldsymbol{L}+\boldsymbol{\omega} \times \hat{\boldsymbol{n}}$ to obtain

$$
\boldsymbol{t}=-p \hat{\boldsymbol{n}}+\left(\mu_{v}+4 \mu / 3\right) \Theta \hat{\boldsymbol{n}}+\mu \boldsymbol{\omega} \times \hat{\boldsymbol{n}}-2 \mu(\Theta \hat{\boldsymbol{n}}-\hat{\boldsymbol{n}} \cdot \boldsymbol{L}) .
$$

Then we use (3.1) to rewrite the dilatation rate in terms of pressure, giving

$$
\boldsymbol{t}=-(1+R) p \hat{\boldsymbol{n}}+\mu \boldsymbol{\omega} \times \hat{\boldsymbol{n}}-2 \mu(\Theta \hat{\boldsymbol{n}}-\hat{\boldsymbol{n}} \cdot \boldsymbol{L}) .
$$

To proceed further we introduce the following differential operators, confined to the directions tangential to the surface, with $h_{i}(i=1,2,3)$ denoting the coordinate scale factors:

$$
\operatorname{grad}_{\|}(\cdot)=\sum_{i=1,2} \frac{\partial(\cdot)}{\partial y_{i}} \otimes \frac{\boldsymbol{e}_{i}}{h_{i}}, \quad \operatorname{div}_{\|}(\cdot)=\sum_{i=1,2} \frac{\partial(\cdot)}{\partial y_{i}} \cdot \frac{\boldsymbol{e}_{i}}{h_{i}}, \quad \operatorname{curl}_{\|}(\cdot)=\sum_{i=1,2} \frac{\boldsymbol{e}_{i}}{h_{i}} \times \frac{\partial(\cdot)}{\partial y_{i}} .
$$

For the last term in (B 2), we first consider the normal component of this vector:

$$
\begin{aligned}
(\Theta \hat{\boldsymbol{n}}-\hat{\boldsymbol{n}} \cdot \boldsymbol{L}) \cdot \hat{\boldsymbol{n}} & =\Theta-\hat{\boldsymbol{n}} \cdot \boldsymbol{L} \cdot \hat{\boldsymbol{n}}=\sum_{i, j} \frac{\boldsymbol{e}_{i}}{h_{i}} \cdot \frac{\partial\left(v_{j} \boldsymbol{e}_{j}\right)}{\partial y_{i}}-\sum_{i, j} \boldsymbol{e}_{3} \cdot \frac{\partial\left(v_{j} \boldsymbol{e}_{j}\right)}{\partial y_{i}} \otimes \frac{\boldsymbol{e}_{i}}{h_{i}} \cdot \boldsymbol{e}_{3} \\
& =\sum_{i, j} \frac{\boldsymbol{e}_{i}}{h_{i}} \cdot \frac{\partial\left(v_{j} \boldsymbol{e}_{j}\right)}{\partial y_{i}}-\sum_{j} \frac{\boldsymbol{e}_{3}}{h_{3}} \cdot \frac{\partial\left(v_{j} \boldsymbol{e}_{j}\right)}{\partial y_{3}}=\operatorname{div}_{\|}(\boldsymbol{v}) .
\end{aligned}
$$

And for the tangential component of $\Theta \hat{\boldsymbol{n}}-\hat{\boldsymbol{n}} \cdot \boldsymbol{L}$ it is possible to write:

$$
\begin{aligned}
\Theta \hat{\boldsymbol{n}}-\hat{\boldsymbol{n}} \cdot \boldsymbol{L}-[(\Theta \hat{\boldsymbol{n}}-\hat{\boldsymbol{n}} \cdot \boldsymbol{L}) \cdot \hat{\boldsymbol{n}}] \hat{\boldsymbol{n}} & =(\hat{\boldsymbol{n}} \cdot \boldsymbol{L} \cdot \hat{\boldsymbol{n}}) \hat{\boldsymbol{n}}-\hat{\boldsymbol{n}} \cdot \boldsymbol{L} \\
& =\operatorname{grad}_{\|}(\hat{\boldsymbol{n}}) \cdot \boldsymbol{v}-\operatorname{grad}_{\|}(\boldsymbol{v} \cdot \hat{\boldsymbol{n}}) \\
& =\operatorname{curl}_{\|}(\boldsymbol{v}) \times \hat{\boldsymbol{n}} .
\end{aligned}
$$

Combining the normal and tangential components of $\Theta \hat{\boldsymbol{n}}-\hat{\boldsymbol{n}} \cdot \boldsymbol{L}$ leads directly to (3.10).

\section{B.2. Derivation of (3.11)}

We begin by noting that (3.4) for the divergence of the viscous stress tensor also applies to the windowed velocity field:

$$
\operatorname{div}[\boldsymbol{\tau}(H \boldsymbol{v})]=\left(\mu_{v}+4 \mu / 3\right) \operatorname{grad}[\Theta(H \boldsymbol{v})]-\mu \operatorname{curl}[\omega(H \boldsymbol{v})] .
$$

As explained in $\S 3.3$, to derive (3.11) one starts with the viscous stress tensor associated with the windowed velocity field given in (3.13). After taking the double divergence of (3.13) and using the expression above, we obtain

$$
\begin{aligned}
\operatorname{div}\{\operatorname{div}[H \boldsymbol{\tau}(\boldsymbol{v})]\}= & \left(\mu_{v}+4 \mu / 3\right) \Delta[\Theta(H \boldsymbol{v})] \\
& -\operatorname{div}\left\{\operatorname{div}\left\{\left[\mu(\boldsymbol{v} \otimes \hat{\boldsymbol{n}}+\hat{\boldsymbol{n}} \otimes \boldsymbol{v})+\left(\mu_{v}-2 \mu / 3\right) \mathbf{1}(\boldsymbol{v} \cdot \hat{\boldsymbol{n}})\right] \delta(f)\right\}\right\} .
\end{aligned}
$$

The windowed equation for the conservation of mass obtained from (3.1) reads

$$
\frac{1}{c_{0}^{2}} \frac{\partial H p}{\partial t}+\rho_{0} \Theta(H \boldsymbol{v})=\boldsymbol{v} \cdot \hat{\boldsymbol{n}} \delta(f)
$$


and can be used to rewrite the dilatation rate in terms of the windowed pressure, giving

$$
\operatorname{div}\{\operatorname{div}[\boldsymbol{\tau}(H \boldsymbol{v})]\}=-R \Delta(H p)-\operatorname{div}\{\operatorname{div}\{[\mu(\boldsymbol{v} \otimes \hat{\boldsymbol{n}}+\hat{\boldsymbol{n}} \otimes \boldsymbol{v})-2 \mu(\boldsymbol{v} \cdot \hat{\boldsymbol{n}}) \mathbf{1}] \delta(f)\}\} .
$$

If we now develop the first divergence in the second term we find

$$
\begin{aligned}
\operatorname{div}\{[ & {[\boldsymbol{v} \otimes \hat{\boldsymbol{n}}+\hat{\boldsymbol{n}} \otimes \boldsymbol{v})-2(\boldsymbol{v} \cdot \hat{\boldsymbol{n}}) \boldsymbol{1}] \delta(f)\} } \\
= & \boldsymbol{v} \operatorname{div}[\hat{\boldsymbol{n}} \delta(f)]+\hat{\boldsymbol{n}} \delta(f) \operatorname{div}(\boldsymbol{v})-\hat{\boldsymbol{n}} \delta(f) \cdot \operatorname{grad}(\boldsymbol{v})-\boldsymbol{v} \cdot \operatorname{grad}[\hat{\boldsymbol{n}} \delta(f)] \\
& -2 \hat{\boldsymbol{n}} \delta(f) \times \boldsymbol{\omega} .
\end{aligned}
$$

Then we can use the vector identity $\operatorname{curl}(\boldsymbol{a} \times \boldsymbol{b})=\operatorname{div}(\boldsymbol{b}) \boldsymbol{a}-\boldsymbol{a} \cdot \operatorname{grad}(\boldsymbol{b})-\operatorname{div}(\boldsymbol{a}) \boldsymbol{b}$ $+\boldsymbol{b} \cdot \operatorname{grad}(\boldsymbol{a})$ and apply the second divergence to arrive at

$$
\begin{aligned}
\operatorname{div}\{\operatorname{div}[\boldsymbol{\tau}(H \boldsymbol{v})]\} & =-R \Delta(H p)-\operatorname{div}\{2 \mu[\operatorname{div}(\boldsymbol{v}) \hat{\boldsymbol{n}}-\hat{\boldsymbol{n}} \cdot \operatorname{grad}(\boldsymbol{v})+\boldsymbol{\omega} \times \hat{\boldsymbol{n}}] \delta(f)\} \\
& =-R \Delta(H p)-\operatorname{div}\left\{2 \mu\left[\Theta \hat{\boldsymbol{n}}-\hat{\boldsymbol{n}} \cdot L^{\mathrm{T}}+\boldsymbol{\omega} \times \hat{\boldsymbol{n}}\right] \delta(f)\right\} .
\end{aligned}
$$

Finally, after using the identity $\hat{\boldsymbol{n}} \cdot \boldsymbol{L}^{\mathrm{T}}=\hat{\boldsymbol{n}} \cdot \boldsymbol{L}+\boldsymbol{\omega} \times \hat{\boldsymbol{n}}$ and the results from appendix B.1 for $\Theta \hat{\boldsymbol{n}}-\hat{\boldsymbol{n}} \cdot \boldsymbol{L}$, we can obtain (3.11).

\section{Appendix C. Solutions for the oscillating plane surface}

By using the mass and momentum equations (3.1) and (3.2) together with the velocity field (4.1) imposed at the plane boundary we can solve for all the variables of interest. In particular we can obtain the following expression for pressure $p$ on the plane boundary:

$$
p\left(x_{1}, x_{2}=0\right)=\rho_{0} c_{0} \frac{k_{1}}{k_{0}} \frac{1+k_{2}^{\prime 2} / k_{1}^{2}}{1+k_{2}^{\prime} k_{2}^{\prime \prime} / k_{1}^{2}} V \mathrm{e}^{\mathrm{i} k_{1} x_{1}-\mathrm{i} \omega t},
$$

where $k_{0}=\omega / c_{0}$ is the lossless acoustic free-field wavenumber, and $k_{2}^{\prime}$ and $k_{2}^{\prime \prime}$ are the normal components of the acoustic and vortical wavenumbers $\kappa^{\prime}$ and $\kappa^{\prime \prime}$, respectively, given by

$$
k_{2}^{\prime}=\sqrt{\kappa^{\prime 2}-k_{1}^{2}}, \quad k_{2}^{\prime \prime}=\sqrt{\kappa^{\prime \prime 2}-k_{1}^{2}} \quad \text { with } \kappa^{\prime 2}=\frac{k_{0}^{2}}{1-4 \mathrm{i} L / 3}, \quad \kappa^{\prime \prime 2}=\frac{\mathrm{i} k_{0}^{2}}{L} .
$$

Similarly we get the following expressions for the normal and tangential components of the viscous surface traction:

$$
\begin{gathered}
\sigma=-2 \mathrm{i} \rho_{0} c_{0} \frac{k_{1}}{k_{0}} L\left(1-\frac{2}{3} \frac{1+k_{2}^{\prime 2} / k_{1}^{2}}{1+k_{2}^{\prime} k_{2}^{\prime \prime} / k_{1}^{2}}\right) V \mathrm{e}^{\mathrm{i} k_{1} x_{1}-\mathrm{i} \omega t}, \\
\tau=-\rho_{0} c_{0} \frac{k_{0} k_{2}^{\prime} / k_{1}^{2}}{1+k_{2}^{\prime} k_{2}^{\prime \prime} / k_{1}^{2}} V \mathrm{e}^{\mathrm{i} k_{1} x_{1}-\mathrm{i} \omega t} .
\end{gathered}
$$

These expressions can be used in (4.2) and (4.3) to evaluate the solution of the CST formulation. The one-dimensional Green's function is defined by

$$
\frac{\partial^{2} G}{\partial x^{2}}+\kappa^{2} G=-\delta(x-y) \quad \text { with } \kappa=k_{20}^{\prime}=\sqrt{k_{0}^{2}-k_{1}^{2}},
$$

together with the appropriate radiation condition at infinity. We can use either the freefield Green's function $G_{\infty}$ or the Neumann Green's function $G_{N}$ defined by requiring 
that $\partial G_{N} / \partial x=0$ at $x=0$. The corresponding expressions are as follows:

$$
G_{\infty}(x \mid y ; \kappa)=\frac{\mathrm{i}}{2 \kappa} \mathrm{e}^{\mathrm{i} \kappa(x-y)}, \quad G_{N}(x \mid y ; \kappa)=\frac{\mathrm{i}}{2 \kappa}\left[\mathrm{e}^{\mathrm{i} \kappa(x-y)}+\mathrm{e}^{-\mathrm{i} \kappa(x+y)}\right] \quad \text { with } x \geqslant y \geqslant 0 .
$$

\section{Appendix D. Solutions for the oscillating cylinder}

This section provides more details on the analytical solution for the test case of a vibrating cylinder in a viscous fluid described in $\S 5.1$. The equations in this section are non-dimensionalized using $r_{0}, \rho_{0}$ and $c_{0}$ as reference values.

\section{D.1. Exact solution}

The velocity field can be expressed as follows:

$$
u(r, \theta, t)=\frac{\partial \varphi}{\partial r}+\frac{1}{r} \frac{\partial \psi}{\partial \theta} \quad \text { and } \quad v(r, \theta, t)=\frac{1}{r} \frac{\partial \varphi}{\partial \theta}-\frac{\partial \psi}{\partial r},
$$

which corresponds to the Helmholtz decomposition of the vector field $v$, with the scalar potential $\varphi$ and the vector potential $\psi \boldsymbol{e}_{z}$ representing the irrotational and vortical components of the velocity, respectively. With the choice of surface velocity given by (5.1), it is possible to infer the azimuthal dependences for the other variables as

$$
(\varphi, p, u, \sigma)=\left(\varphi_{m}, p_{m}, u_{m}, \sigma_{m}\right) \cos (m \theta) \mathrm{e}^{-\mathrm{i} \omega t}, \quad(\psi, v, \tau)=\left(\psi_{m}, v_{m}, \tau_{m}\right) \sin (m \theta) \mathrm{e}^{-\mathrm{i} \omega t},
$$

where $\sigma$ and $\tau$ denote the total normal and tangential stresses on $r=$ constant. Solutions for other quantities of interest can then be written in terms of the scalar potentials:

$$
\begin{gathered}
p_{m}=\mathrm{i} K \varphi_{m}+\frac{4}{3} \frac{K}{S}\left(\frac{\mathrm{d}^{2}}{\mathrm{~d} r^{2}}+\frac{1}{r} \frac{\mathrm{d}}{\mathrm{d} r}-\frac{m^{2}}{r^{2}}\right) \varphi_{m}, \\
\sigma_{m}=-\mathrm{i} K \varphi_{m}-2 \frac{K}{S}\left[\left(\frac{1}{r} \frac{\mathrm{d}}{\mathrm{d} r}-\frac{m^{2}}{r^{2}}\right) \varphi_{m}-m \frac{\mathrm{d}}{\mathrm{d} r}\left(\frac{1}{r} \psi_{m}\right)\right], \\
\tau_{m}=\mathrm{i} K \psi_{m}+2 \frac{K}{S}\left[\left(\frac{1}{r} \frac{\mathrm{d}}{\mathrm{d} r}-\frac{m^{2}}{r^{2}}\right) \psi_{m}-m \frac{\mathrm{d}}{\mathrm{d} r}\left(\frac{1}{r} \varphi_{m}\right)\right] .
\end{gathered}
$$

The linearized Navier-Stokes equations (3.1)-(3.3) can be expressed as two uncoupled Bessel equations for the potentials:

$$
\begin{gathered}
\frac{\mathrm{d}^{2} \varphi_{m}}{\mathrm{~d} r^{2}}+\frac{1}{r} \frac{\mathrm{d} \varphi_{m}}{\mathrm{~d} r}-\frac{m^{2}}{r^{2}} \varphi_{m}+\frac{K^{2}}{1+\hat{R}} \varphi_{m}=0, \\
\frac{\mathrm{d}^{2} \psi_{m}}{\mathrm{~d} r^{2}}+\frac{1}{r} \frac{\mathrm{d} \psi_{m}}{\mathrm{~d} r}-\frac{m^{2}}{r^{2}} \psi_{m}+\mathrm{i} S \psi_{m}=0 .
\end{gathered}
$$

In addition, we require that both $\varphi_{m}(r)$ and $\psi_{m}(r)$ behave as decaying outgoing waves in the far field $(r \rightarrow \infty)$. Therefore, the solutions are directly given by Hankel functions of the first kind:

$$
\varphi_{m}(r)=A_{m} \mathrm{H}_{m}\left(K^{\prime} r\right), \quad \psi_{m}(r)=B_{m} \mathrm{H}_{m}\left(K^{\prime \prime} r\right),
$$

where $K^{\prime}=K / \sqrt{1+\hat{R}}$ and $K^{\prime \prime}=\sqrt{\mathrm{i} S}$ are the radial wavenumbers for the acoustic and vorticity waves, respectively. The amplitudes $A_{m}$ and $B_{m}$ of these waves are specified 
by the no-slip boundary conditions at the cylinder wall $r=1$ :

$$
\frac{\mathrm{d} \varphi_{m}}{\mathrm{~d} r}+\frac{m}{r} \psi_{m}=U_{m}, \quad \frac{\mathrm{d} \psi_{m}}{\mathrm{~d} r}+\frac{m}{r} \varphi_{m}=-V_{m} .
$$

If we consider a unit normal forcing $\left(U_{m}=1\right.$ and $\left.V_{m}=0\right)$ we have

$$
\begin{gathered}
\frac{1}{A_{m}}=\frac{S L}{1+\hat{R}} \mathrm{H}_{m}^{\prime}\left(K^{\prime}\right)-\frac{(-\mathrm{i} L)^{1 / 2} m^{2} \mathrm{H}_{m}\left(K^{\prime \prime}\right) \mathrm{H}_{m}\left(K^{\prime}\right)}{\mathrm{H}_{m}^{\prime}\left(K^{\prime \prime}\right)}, \\
B_{m}=-\frac{m A_{m} \mathrm{H}_{m}\left(K^{\prime}\right)}{K^{\prime \prime} \mathrm{H}_{m}^{\prime}\left(K^{\prime \prime}\right)} .
\end{gathered}
$$

Alternatively, if we consider a unit tangential 'dolphin-skin' forcing $\left(U_{m}=0\right.$ and $\left.V_{m}=1\right)$ the coefficients are given by

$$
\begin{gathered}
A_{m}=-\frac{m B_{m} \mathrm{H}_{m}\left(K^{\prime \prime}\right)}{K^{\prime} \mathrm{H}_{m}^{\prime}\left(K^{\prime}\right)}, \\
\frac{1}{B_{m}}=\frac{(1+\hat{R})^{1 / 2} m^{2} \mathrm{H}_{m}\left(K^{\prime \prime}\right) \mathrm{H}_{m}\left(K^{\prime}\right)}{\mathrm{H}_{m}^{\prime}\left(K^{\prime}\right)}-(\mathrm{i} L)^{1 / 2} S \mathrm{H}_{m}^{\prime}\left(K^{\prime \prime}\right) .
\end{gathered}
$$

\section{D.2. Solutions for the acoustic analogies}

The prediction of the Curle-Lighthill analogy for the test case can be calculated by using (D 2) to write

$$
\left[\frac{1}{r} \frac{\mathrm{d}}{\mathrm{d} r}\left(r \frac{\mathrm{d}}{\mathrm{d} r}\right)-\frac{m^{2}}{r^{2}}+K^{2}\right]\left(H p_{m}\right)=-q_{m} \delta\left(r-r_{0}\right)+\frac{1}{r} \frac{\mathrm{d}}{\mathrm{d} r}\left[d_{m} r \delta\left(r-r_{0}\right)\right],
$$

where $d_{m}=-\sigma_{m}(1)$ represents the strength of the normal dipole while $q_{m}=-\mathrm{i} K U_{m}+$ $m \tau_{m}(1)$ is an equivalent surface monopole, representing the normal acceleration and the tangential stress on the surface. These expressions for $d_{m}$ and $q_{m}$ are directly obtained from (3.9). Note that the volume term is omitted in (D 14).

The solution to (D 14) can be written as

$$
p_{m}(r)=q_{m} G_{m}(r \mid 1 ; K)+d_{m}\left[\frac{\partial G_{m}(r \mid a ; K)}{\partial a}\right]_{a=1},
$$

with $r \geqslant 1$ and the Green's functions being defined by

$$
\left[\frac{1}{r} \frac{\mathrm{d}}{\mathrm{d} r}\left(r \frac{\mathrm{d}}{\mathrm{d} r}\right)-\frac{m^{2}}{r^{2}}+K^{2}\right] G_{m}(r \mid a ; K)=-\frac{1}{a} \delta(r-a) .
$$

As explained in $\S 3.4$, (D 14) is a generalized Helmholtz-type equation valid in all space, which can be solved using any causal Green's function of the operator on the left. We use either the free-field Green's function $G_{m, \infty}$ or the Neumann Green's function $G_{m, N}$ that satisfies the hard-wall boundary condition $\partial G_{m, N} / \partial r=0$ at $r=1$. The corresponding expressions are, for $r \geqslant a \geqslant 1$,

$$
G_{m, \infty}(r \mid a ; K)=\frac{\mathrm{i} \pi}{2} \mathrm{~J}_{m}(K a) \mathrm{H}_{m}(K r), \quad G_{m, N}(r \mid a ; K)=\frac{-1}{K a} \frac{\mathrm{H}_{m}(K r)}{\mathrm{H}_{m}^{\prime}(K a)} .
$$


For the analogy based on the viscous wave equation (3.7), the equation for the modal pressure $p_{m}$ is

$$
\begin{gathered}
\left\{(1+\hat{R})\left[\frac{1}{r} \frac{\mathrm{d}}{\mathrm{d} r}\left(r \frac{\mathrm{d}}{\mathrm{d} r}\right)-\frac{m^{2}}{r^{2}}\right]+K^{2}\right\}\left(H p_{m}\right) \\
=-q_{m} \delta\left(r-r_{0}\right)+\frac{1}{r} \frac{\mathrm{d}}{\mathrm{d} r}\left[d_{m} r \delta\left(r-r_{0}\right)\right],
\end{gathered}
$$

with the source terms given by $d_{m}=(1+R) p_{m}(1)$ and $q_{m}=-\mathrm{i} K U_{m}+\mathrm{i} K m \psi_{m}(1)$. The solution is formally the same as in (D 15):

$$
p_{m}(r)=q_{m} \hat{G}_{m}\left(r \mid 1 ; K^{\prime}\right)+d_{m}\left[\frac{\partial \hat{G}_{m}\left(r \mid a ; K^{\prime}\right)}{\partial a}\right]_{a=1},
$$

but with the difference that one uses the Green's function $\hat{G}_{m}$ for the viscous wave operator in (D 18), which can be written as

$$
\hat{G}_{m}\left(r \mid a ; K^{\prime}\right)=\frac{1}{1+\hat{R}} G_{m}\left(r \mid a ; K^{\prime}\right),
$$

where $G_{m}$ can be either the free-field or Neumann Green's function. It can be shown that the ratio $\hat{G}_{m}\left(1 \mid 1 ; K^{\prime}\right) / G_{m}(1 \mid 1 ; K)$ tends to $(1+\hat{R})^{-1 / 2}$ for small Helmholtz numbers $K$.

\section{REFERENCES}

Crighton, D. G. 1975 Basic principles of aerodynamic noise generation. Prog. Aerosp. Sci. 16 (1), 31-96.

Curle, N. 1955 The influence of solid boundaries on aerodynamic sound. Proc. R. Soc. Lond. 231, $505-514$.

DAVIS, A. M. J. \& NAGEM, R. J. 2006 Curle's equation and acoustic scattering by a sphere. J. Acoust. Soc. Am. 119 (4), 2018-2026.

Dishington, R. H. 1965 Rate of surface-strain tensor. Am. J. Phys. 33, 827-831.

FARASSAT, F. 1994 Introduction to generalized functions with applications in aerodynamics and aeroacoustics. NASA Technical Paper 3428.

Ffowcs Williams, J. E. \& HaWkings, D. L. 1969 Sound generation by turbulence and surfaces in arbitrary motion. Phil. Trans. R. Soc. Lond. A 264, 321-342.

Goldstein, M. E. 1976 Aeroacoustics. McGraw-Hill.

IRGENS, F. 2008 Continuum Mechanics. Springer.

Lighthill, M. J. 1952 On sound generated aerodynamically I. General theory. Proc. R. Soc. Lond. 211, 564-587.

Morfey, C. L., Powles, C. J. \& Wright, M. C. M. 2011 Green's function in computational aeroacoustics. Intl J. Aeroacoust. 10, 117-160.

Morse, P. M. \& Ingard, K. U. 1968 Theoretical Acoustics. McGraw-Hill.

Pierce, A. D. 1989 Acoustics: An Introduction to its Physical Principles and Applications. Acoustical Society of America.

SHARIFF, K. \& WANG, M. 2005 A numerical experiment to determine whether surface shear-stress fluctuations are a true sound source. Phys. Fluids 17, 107-105.

Wu, J.-Z., MA, H.-Y. \& ZHou, M.-D. 2006 Vorticity and Vortex Dynamics. Springer. 